\title{
Special issue on microbubbles: from contrast enhancement to cancer therapy
}

\author{
Eleanor Stride $\cdot$ Mohan Edirisinghe
}

Received: 24 June 2009/Accepted: 24 June 2009/Published online: 28 July 2009

(C) International Federation for Medical and Biological Engineering 2009

The clinical use of microbubbles originated with the fortuitous discovery by Joyner that intravenous injection of certain liquids produced contrast enhancement in ultrasound images [3]. Subsequent investigation revealed that it was not the liquids themselves which were responsible for the observed effect, but rather the presence of gas microbubbles either suspended in the liquid or formed at the tip of the catheter through which it was being injected. Since then, the use of microbubbles in diagnostic and, more recently, therapeutic applications has become a rapidly expanding field of research. The aim of this special issue is to provide an up-to-date overview of the developments in the physics, engineering and clinical applications of microbubbles.

Microbubble ultrasound contrast agents have now been in clinical use for more than two decades and their evolving applications are discussed in detail in the comprehensive review by Cosgrove and Harvey [1] who have extensive clinical experience in this area. The primary application of microbubbles is in echocardiography, for ventricular opacification and delineation of endocardial borders. They have also been used successfully for the assessment of systolic function and left ventricular volume, and for identifying myocardial infarction and coronary artery stenoses. As explained in the paper, they are moreover becoming rapidly accepted for the detection, characterisation and image guided treatment of focal liver lesions. They have also been used for functional investigations in other organs including the kidneys, spleen and pancreas and for assessment of disease in the bowel, prostate, breast

E. Stride $(\bowtie) \cdot$ M. Edirisinghe

Department of Mechanical Engineering, University College

London, Torrington Place, London WC1E 7JE, UK

e-mail: e_stride@meng.ucl.ac.uk and lymph nodes as well as non-vascular applications, such as assessment of fallopian tube patency and detection of ureteric reflux.

It is the highly non-linear response of microbubbles to ultrasound that has facilitated the development of a range of contrast-specific imaging protocols which have greatly increased the diagnostic potential of ultrasound imaging. For example, in combination with advances in threedimensional visualisation techniques, harmonic imaging with microbubbles has enabled mapping of the microcirculation for the characterisation of tumour vascularity, and also in the brain as described in the paper by Meairs et al. [5]. To fully exploit the potential of microbubbles, however, it is important to understand the underlying physics of their behaviour and there has consequently been intensive research conducted into their theoretical and experimental characterisation. These studies are reviewed in the paper by de Jong et al. [2], who together with Versluis were part of the team at the Universities of Rotterdam and Twente in The Netherlands responsible for the development of a highspeed camera capable of imaging at 25 million frames/s for direct observation of microbubble oscillations.

Microbubbles have also shown great potential in quantitative and targeted (molecular) imaging. As discussed in the paper by Tortoli et al. [8] they are widely used in Doppler studies, especially in the microcirculation, to improve the signal-to-noise ratio. Interpretation of the microbubble signals can however be complicated not only by their non-linearity, but also by a variety of phenomena including destruction, deflation and displacement of microbubbles due to radiation force. The aim of the work by Tortoli et al. has been to assess the relative significance of these effects for the accuracy of clinical measurements and to gain better understanding of the changes in microbubble characteristics over time. 
The ability to attach molecules to microbubbles that are targeted to specific vascular receptor sites has opened up further opportunities for tissue-specific imaging, and conditions currently under investigation include inflammation, angiogenesis and atherosclerosis. It also has important implications for therapeutic applications. In drug and gene delivery, for example, microbubbles can be used as targeted vehicles which are loaded with the required therapeutic agent, traced to the target site using low-intensity ultrasound and then, once attached, destroyed with a highintensity burst to release the material locally, thus avoiding systemic administration, e.g. of toxic chemotherapy. The preparation of targeted microbubbles is discussed in detail in the paper by Klibanov et al. [4] who have developed new strategies for more rapid, stronger attachment of microbubbles under high shear which to date has posed a considerable challenge to successful targeting of microbubbles in vivo.

As described by Meairs et al. [5], there is also substantial evidence that motion of the microbubbles increases the permeability of both individual cell membranes and the endothelium including temporary opening of the bloodbrain barrier. Thus bubbles can act not only as delivery vehicles, but also actively promote uptake of therapeutic material. As Meairs et al. discuss, minimally invasive drug delivery to the brain represents a significant clinical challenge and hence the positive results from studies with microbubbles are extremely encouraging. Ongoing work to determine the mechanisms and potential side effects of microbubble-mediated delivery are urgently required to facilitate the development of protocols acceptable for mainstream clinical practice.

Yoshizawa et al. [9] discuss another related area which has been the subject of much research and that is the use of cavitation in high-intensity focused ultrasound (HIFU) surgery, in order to increase the speed and efficacy of treatment. Their study focuses on HIFU lithotripsy and the use of dual-frequency ultrasound to create and control a cavitation cloud which is made to collapse so that the simultaneous impact of the bubbles produces stone fragmentation. In their experiments, Yoshizawa et al. demonstrate the advantages of the dual-frequency technique over single-frequency excitation and the relationship between the acoustic emissions from the cavitation cloud and the extent of stone erosion which offers a method for treatment monitoring.

Applications such as HIFU lithotripsy exploit the ability of microbubbles to cause significant tissue damage. In both cases, the damage occurs as a result of violent bubble oscillations which are deliberately generated over periods that are very long compared with typical diagnostic exposures. Nevertheless, and despite considerable investigation, there remain some concerns over the safety of microbubbles under diagnostic conditions. These were highlighted in 2007 by the imposition of a "black-box" warning on two commercially available ultrasound contrast agents by the USA Food and Drug Administration (FDA). The restrictions have since been relaxed but the incident has stimulated further investigation of microbubble safety which is reviewed in the paper by ter Haar [7] together with the recommendations from European and International Bodies concerned with safety in clinical ultrasound.

Concerns over safety and developments in diagnostic and therapeutic applications have greatly increased the need for more advanced preparation technologies which provide a high degree of control over microbubble characteristics. For drug delivery, for example, a welldefined microbubble size distribution, uniform drug content and coating destruction threshold are critical in order to ensure accurate dosing. Similarly, as more advanced imaging techniques are developed which exploit the complex non-linear features of the microbubble signal and/or enable quantification of tissue perfusion, the ability to predetermine the acoustic response of a microbubble suspension is becoming increasingly important. In their paper, Stride and Edirisinghe [6] compare conventional techniques for microbubble preparation with two new methods they have been investigating based on electrohydrodynamic and microfluidic processing, respectively. Both new methods enable microbubbles to be prepared in a single step with a predetermined mean diameter and narrow size distribution and scaling up of the technology to produce industrially relevant yields and are currently under investigation.

In summary, as the eight papers in this special issue demonstrate, microbubbles have already revolutionised ultrasound imaging and show enormous potential for therapeutic applications. In order to fully realise this potential, it is essential that the basic physics of microbubbles and in particular their interaction with tissue are fully understood; and that the technology is developed to produce microbubbles with well-defined characteristics, that are stable and have the capacity to be targeted to specific sites in vivo. As the work described here shows, there is a wealth of exciting and innovative research currently in progress to address these challenges.

\section{References}

1. Cosgrove D, Harvey C (2009) Clinical use of microbubbles for imaging and therapy. Med Biol Eng Comput 47. doi: 10.1007/s11517-009-0434-3

2. de Jong N, Emmer M, van Wamel A, Versluis M (2009) Ultrasonic characterization of ultrasound contrast agents. Med Biol Eng Comput 47. doi:10.1007/s11517-009-0497-1 
3. Gramiak R, Shah P (1968) Echocardiography of the aortic root. Invest Radiol 3:356-366

4. Klibanov A (2009) Preparation of targeted microbubbles: ultrasound contrast agents for molecular imaging. Med Biol Eng Comput 47. doi:10.1007/s11517-009-0498-0

5. Meairs S, Alonso A, Fatar M, Kern R, Hennerici M (2009) Microbubbles traversing the blood brain barrier for imaging and therapy. Med Biol Eng Comput 47. doi:10.1007/s11517-0090468-64

6. Stride E, Edirisinghe M (2009) Novel preparation techniques for controlling microbubble uniformity—a comparison. Med Biol Eng Comput 47. doi:10.1007/s11517-009-0490-8
7. ter Haar G (2009) Safety and bio-effects of ultrasound contrast agents. Med Biol Eng Comput 47

8. Tortoli P, Guidi F, Mori F, Vos, H (2009) The use of microbubbles in Doppler ultrasound studies. Med Biol Eng Comput 47. doi: 10.1007/s11517-008-0423-y

9. Yoshizawa S, Ikeda T, Ito A, Ota R, Takagi S, Matsumoto $Y$ (2009) High intensity focused ultrasound lithotripsy with cavitating microbubbles. Med Biol Eng Comput 47. doi:10.1007/ s11517-009-0471-y 